\title{
Correlation of Blood Lipid Profile, Blood Lipid Ratio and Homocysteine in Patients with Hypothyroidism
}

\author{
Hou Zhenjiang ${ }^{1}{ }^{*}$, Mu Zhaoxin ${ }^{1}$, Zhang Jingyu ${ }^{2}$, Fan Hong ${ }^{2}$, Hou Jianzhang ${ }^{3}$, Wang Hongsheng ${ }^{2}$ \\ ${ }^{1}$ Institute of Thyroid Diseases, Cangzhou Medical College, Cangzhou, China \\ ${ }^{2}$ Laboratory Diagnostics Division, Hebei Cangzhou Hospital of Integrated Traditional Chinese and Western Medicine, Cangzhou, China \\ ${ }^{3}$ Department of Surgery, Cangzhou People's Hospital, Cangzhou Medical College, Cangzhou, China
}

Email address:

houzhenjiang@sina.com (Hou Zhenjiang)

${ }^{*}$ Corresponding author

\section{To cite this article:}

Hou Zhenjiang, Mu Zhaoxin, Zhang Jingyu, Fan Hong, Hou Jianzhang, Wang Hongsheng. Correlation of Blood Lipid Profile, Blood Lipid Ratio and Homocysteine in Patients with Hypothyroidism. Clinical Medicine Research. Vol. 6, No. 3, 2017, pp. 106-110.

doi: $10.11648 /$ j.cmr.20170603.18

Received: March 23, 2017; Accepted: April 27, 2017; Published: May 4, 2017

\begin{abstract}
To investigate the correlation between hypothyroidism and subclinical hypothyroidism in serum thyroid hormones, blood lipid profile, blood lipid ratio and homocysteine, the authors calculated the ratio of blood lipid and carried on statistical analysis through measuring the level of serum thyroid hormones, blood lipid, homocysteine among 60 patients with hypothyroidism, 140 patients with subclinical hypothyroidism and 60 controls. As a result, firstly, the levels of serum TSH in hypothyroidism group and subclinical hypothyroidism group were significantly higher than those in the control group $(P$ $<0.001)$. The difference between the subclinical hypothyroidism group and the control group had statistical significance $(P$ $<0.05$ ). What's more, the patients, who were hypothyroidism, were significantly lower than those in the control group in the levels of serum FT3 and FT4. Moreover, compared with the hypothyroidism and subclinical hypothyroidism, on the one hand, the levels of serum FT3 and FT4 obviously decreased, on the other hand, the levels of serum TSH and Hcy were significantly increased $(P<0.01)$. As to the correlation of thyroid hormones with blood lipid ratio and non-HDL-C, TC showed the highest correlation and the sequences were non-HDL-C, LDL-C and LCI. In the end, the authors came to the conclusion that the detection of serum TSH, blood lipid ratio and Hcy were of great value in the transformation from subclinical hypothyroidism to hypothyroidism and the prediction of hypothyroidism and the cause of cardiovascular diseases.
\end{abstract}

Keywords: Hypothyroidism, Subclinical Hypothyroidism, Thyroid Hormone, Blood Lipid Ratio, Homocysteine

\section{Introduction}

As a common endocrine system disease, hypothyroidism includes a group of syndromes due to decreasing synthesis and secretion of thyroid hormones and insufficient biological effects. Subclinical hypothyroidism $(\mathrm{SCH})$ refers to increased level of serum thyroid stimulating hormone (TSH) while the free thyroid hormones remain at normal levels [1] in the absence of clinical symptoms or showing mild symptoms; the diagnosis of SCH must be based on serological examinations. $\mathrm{SCH}$ prevalence in humans reaches up from $5 \%$ to $15 \%$, and gradually increases with age growth. For women over the age of 60 , the prevalence may be up to $20 \%$ [2]. It is estimated that about $2 \%$ to $5 \%$ of SCH patients transforming to clinical hypothyroidism each year. With elevated serum TSH levels in $\mathrm{SCH}$ patients, the incidence of cardiovascular diseases may increase greatly [3]. Studies have shown that plasma homocysteine (Hcy) levels and the occurrence and development of coronary atherosclerosis are close correlation; high Hcy in patients with hypothyroidism implies a higher incidence of cardiovascular diseases, especially closely correlated with AS [4]. The levels of serum TSH, FT3, FT4, T3 and T4, blood lipid and Hcy are measured and blood lipid ratio is calculated for relevant analysis for the purpose of the prevention and treatment of thyroid disorders and other related diseases. It is hereby reported as follows. 


\section{Data and Methods}

\subsection{General Data}

A total of 200 patients with hypothyroidism visiting our hospital health examination and our Endocrinology Dept. for treatment from April 2015 to September 2016, who were include as the study subject. Among the subjects, 60 patients were in the hypothyroidism group: 21 males and 39 females, aging from 20 to 73 years old with mean age of $(49.31 \pm 15.28)$ years old; 140 patients were in the SCH group: 42 males and 98 females, aging from 26 to 77 years old with mean age of $(48.63 \pm 14.92)$ years old; and 60 healthy subjects from health examination at the same period were treated as the control group: 25 males and 35 females aging from 20 to 82 years with mean age of $(45.18 \pm 13.26)$ years old. There was no significant difference in sex and age among the three groups $(\chi 2=2.964, P=0.578$ VS $\chi 2=0.843, P=0.512)$. The following were excluded: with a history of liver and kidney diseases, hypertension and coronary atherosclerotic heart disease (CHD), dyslipidemia of other causes. And no medication that may impact thyroid functions and blood lipids were taken in the recent three months.

\subsection{Methods}

\subsubsection{Detection Methods}

All subjects were fasting for more than $12 \mathrm{~h} .6 \mathrm{ml}$ of venous blood was taken during 7:30 to 9:30 in the morning and dispensed in 2 packs. The serum was separated centrifugally at $4000 \mathrm{r} / \mathrm{min}$ in time. Thyroid stimulating hormone (TSH), total triiodothyronine (TT3), total thyroxine (TT4), free triiodothyronine (FT3) and free thyroxine (FT4) were measured with Gobas E601 Immune Chemiluminescence Apparatus provided by German Roche Limited; and agents used were originally provided by Roche. Three acyl glycerol (TG), total cholesterol (TC), high density lipoprotein cholesterol (HDL-C) and low intensity lipoprotein cholesterol (LDL-C) were measured with HITACHI-020 Automatic Biochemical Analyzer. Blood lipid ratios (TC/HDL-C, TG/HDL-C and LDL-C/HDL-C), lipid comprehensive index ( $\mathrm{LCI}=\mathrm{TC} \times \mathrm{TG} \times \mathrm{LDL}-\mathrm{C} / \mathrm{HDL}-\mathrm{C})$ and non-HDL-C ( = TC-HDL

-C) were calculated. Homocysteine (Hcy) was measured with AXSYM Automatic Rapid Immunoassay provided by Abbott USA.

\subsubsection{Diagnostic Criteria Hypothyroidism}

Presence of hypothyroidism symptoms or signs; TSH> $4.20 \mathrm{mIU} / \mathrm{L}$; and FT4 < $12 \mathrm{pmol} / \mathrm{L}$ and/or FT3 $<3.10 \mathrm{pmol} / \mathrm{L}$. $\mathrm{SCH}$ : no presence of hypothyroidism symptoms or signs; TSH> $4.20 \mathrm{mIU} / \mathrm{L}$; and normal levels of FT3 and FT4.

\subsection{Statistical Analysis}

SPSS 18.0 statistical software was used for data processing. Measurement data was presented in mean \pm standard deviation $(\bar{x} \pm \mathrm{s})$. One-way analysis of variance was used for comparison. Linear correlation analysis was used for the correlation of thyroid hormones with blood lipid profile and Hcy. $\mathrm{P}<0.05$ denoted significant difference.

\section{Results}

\subsection{Comparison of Thyroid Hormone Levels in Each Group}

The levels of serum TSH in patients with hypothyroidism were significantly higher than those in the $\mathrm{SCH}$ and control groups $(P<0.01)$; and the levels of serum FT3 and FT4 were lower than those in the SCH and control groups. There was no significant difference regarding the levels of serum FT3 and FT4 between the SCH and control groups $(P>0.05)$. The serum levels of T3 and T4 in the hypothyroidism and $\mathrm{SCH}$ groups were lower than those in the control group, but the difference was not statistically significant $(P>0.05)$. The results are shown in Table 1.

Table 1. Comparison of thyroid hormone levels in each group $(\bar{x} \pm \mathrm{s})$.

\begin{tabular}{llllll}
\hline Groups & n & TSH(mIU/L) & FT3(pmol/L) & FT4(pmol/L) & T3(pmol/L) \\
\hline Hypothyroidism & 60 & $32.7 \pm 12.58^{* *}$ & $3.57 \pm 1.11^{*}$ & $8.175 \pm 3.210^{* *}$ & $1.65 \pm 0.53$ \\
Subclinical hypothyroidism & 140 & $8.679 \pm 2.581 *$ & $4.60 \pm 1.13$ & $16.57 \pm 12.18$ & $1.75 \pm 0.62$ \\
controls & 60 & $2.308 \pm 1.023$ & $4.85 \pm 0.65$ & $18.59 \pm 7.04$ & $1.88 \pm 0.34$ \\
\hline
\end{tabular}

Note: Compared with the normal control group, ${ }^{*} P<0.05$, ** $P<0.01$.

\subsection{Comparison of Blood Lipid Profile and Hcy Levels in Each Group}

The levels of serum TC, TG, LDL-D and Hcy in patients with hypothyroidism were significantly higher than those in the $\mathrm{SCH}$ and control groups $(P<0.05)$. There was no significant difference regarding the levels of TC, TG, HDL-D and LDL-C between the SCH and control groups $(P>0.05)$. The level of Hcy in the SCH group was also higher than that in the control group $(P<0.05)$. The results are shown in Table 2 .

Table 2. Comparison of blood lipid profile (mmol/L) and Hcy levels in each group $(\bar{x} \pm \mathrm{s})$.

\begin{tabular}{llllll}
\hline Groups & $\mathbf{n}$ & TC & TG & HDL-C & LDL-C \\
\hline Hypothyroidism & 60 & $5.424 \pm 1.61 *$ & $1.97 \pm 1.57 *$ & $1.159 \pm 0.42 *$ & $3.90 \pm 1.14 *$ \\
Subclinical hypothyroidism & 140 & $5.029 \pm 1.112$ & $1.650 \pm 1.148$ & $1.255 \pm 0.335$ & $3.326 \pm 0.916$ \\
controls & 60 & $4.967 \pm 0.96$ & $1.577 \pm 1.200$ & $1.365 \pm 0.326$ & $3.129 \pm 0.380$ \\
\hline
\end{tabular}

Note: Compared with the normal control group, ${ }^{*} P<0.05, * * P<0.01$. 


\subsection{Comparison of Blood Lipid Ratios and Non-HDL-C in Each Group}

The levels of TG/HDL-C, LDL-C/HDL-C, LCI and non-HDL-C in patients with hypothyroidism were significantly higher than those in the $\mathrm{SCH}$ and control groups
$(P<0.05)$. In addition to the higher LDL-C/HDL-C and non-HDL-C in the SCH group than those in the control group, there was no significant difference regarding other blood lipid ratios between the $\mathrm{SCH}$ and control groups $(P>0.05)$. The results are shown in Table 3 .

Table 3. Comparison of blood lipid ratios and non-HDL-C in each group.

\begin{tabular}{lllllll}
\hline Groups & n & TC/HDL-C & TG/HDL-C & LDL-C/HDL-C & LCI & non-HDL-C \\
\hline Hypothyroidism & 60 & $3.89 \pm 1.17$ & $1.53 \pm 1.41 *$ & $2.90 \pm 0.93 *$ & $33.92 \pm 47.21 * *$ & $4.04 \pm 1.41 *$ \\
Subclinical hypothyroidism & 140 & $3.872 \pm 1.047$ & $1.390 \pm 1.258$ & $2.63 \pm 0.872 *$ & $24.354 \pm 28.038$ & $3.866 \pm 1.07 *$ \\
controls & 60 & $3.804 \pm 1.017$ & $1.320 \pm 1.314$ & $2.412 \pm 0.824$ & $23.432 \pm 29.408$ \\
\hline
\end{tabular}

Note: Compared with the normal control group, ${ }^{*} P<0.05$, $* * P<0.01$.

\subsection{Correlation Analysis among Thyroid Hormones and Blood Lipid Profile, Blood Lipid Ratios, Non-HDL-C and Hcy}

TSH positively correlated with TC, TG, HDL-D, LDL-C, TC/HDL-C, TG/HDL-C, LDL-C/HDL-C, LCI and non-HDL -C. In addition to $P=0.007$ for TG, $P<0.001$ for the remaining. FT3, FT4, T3 and T4 negatively correlated with TC, TG, HDL-D, LDL-D, TC/HDL-C, TG/HDL-C, LDL-C/HDL-C, LCI and non-HDL-C. In addition to $P=0.03$ and 0.007 for TG/HDL-C with T3 and T4, respectively, $P<0.001$ for the remaining. Comprehensive analysis showed that as to the correlation of TH with blood lipid ratio, non-HDL-C and Hcy, TC showed the highest correlation followed by non-HDL-C, LDL- C and LCI.

\section{Discussions}

Since Mason et al [5] first reported Blood Cholesterol Values in Hyperthyroidism and Hypothyroidism Their Significance in NEJM in 1930, the importance of studies have been highly attached to the relationship between hypothyroidism and lipid metabolism. Garduno GJ et al [6] found that the normal higher TSH and lower TH levels associated with blood lipids and positively correlated with TC, LDL-C and TG, and negatively correlated with HDL-C, with the increase of TSH [7]. Li Fang [8] found that patients with hypothyroidism had significantly higher TG, TC, and LDL-C than those in the control group, and $\mathrm{SCH}$ patients mainly had higher LDL-C and TC. Song Qingzhang et al [9] reported that patients with hypothyroidism had significantly higher TG, TC, and LDL-C than those in the control group and had significantly lower HDL-C than those in the control group. Toruner et al [10] found that $\mathrm{SCH}$ patients, regardless of $\mathrm{TSH} \geq 10 \mathrm{mIU} / \mathrm{mL}$ or $<10 \mathrm{mIU} / \mathrm{mL}$, had significantly higher plasma TC, TG and LDL-C than those in the control group. LópezRubio et al [11] conducted a correlation analysis regarding $\mathrm{TH}$ and blood lipids in $326 \mathrm{SCH}$ patients and found that SCH correlated to with high TG. Van Tienhoven-wind et al $[12,13]$ reported that $\mathrm{SCH}$ patients had significantly higher TC, TG and LDL-C. Laway [14] found that SCH patients had significantly higher TC and TG than those in the control group. In this study, it is found that patients with hypothyroidism had significantly higher TC, TG and LDL-C than those in the $\mathrm{SCH}$ and control groups $(P<0.05)$ and decrease of HDL-C; the levels of TC, TG, HDL-C and LDL-C in the $\mathrm{SCH}$ group changed accordingly comparing with the hypothyroidism group but there was no significant difference between the two groups $(P>0.05)$. It was consistent with the report of Díez et al [15] that there was no significant difference regarding serum TC, TG, LDL-C, and HDL-C between SCH patients and people with normal thyroid functions. Therefore, further studies required to discuss the relationship between $\mathrm{SCH}$ patients and dyslipidemia.

Blood lipid determination is a common indicator for clinical evaluation of the risk and prognosis of CHD. However, not all CHD patients undergo significant changes in all of their lipid-related levels. LDL-C/HDL-C may serve as an independent predictor for myocardial infarction. TC/HDL-C and LDL-C/HDL-C are more sensitive to CHD diagnosis than a single indicator [16]. TC/HDL-C and LDL-C/HDL-C can accurately reflect the comprehensive level of in vivo lipid metabolism and the risk of CHD. Compared with TC/HDL-C, LDL-C/HDL-C is of more value in predicting carotid atherosclerotic plaque [17]. Xie Yinjun [18] reported that LCI in the CHD group was significantly higher than that in the control group and LCI in the unstable angina pectoris (UAP) group and acute myocardial infarction (AMI) group was higher than that in the stable angina pectoris (SAP) group $(P<0.05)$; and there was no significant difference in the LCI between the UPA and AMI groups; therefore LCI was believed to be a good indicator of the risk of CHD. Non-HDL-C represented the total cholesterol causing atherosclerosis and might accurately reflect the cholesterol levels of atherosclerotic lipoprotein granules; its role in prediction and prognosis of CHD was significantly better than LDL-C [19]. Chai Yuqiong et al [20] found that non-HDL-C could be used as a supplement to LDL-C and served as a new indicator to assess the severity of coronary artery disease, recurrence risk of cardiovascular events, and residual risk of cardiovascular events. The results of this study showed that patients with hypothyroidism had significantly higher TG/HDL-C, LDL-C/HDL-C, LCI and non-HDL-C than those in the $\mathrm{SCH}$ and control groups; the $\mathrm{SCH}$ group had higher LDL-C/HDL-C and non-HDL-C than 
the control group and there was no significantly difference in the remaining indicators between the two groups. As to the correlation of $\mathrm{TH}$ with blood lipid ratios, TC showed the highest correlation followed by non-HDL-C, LDL-C and LCI. TG/HDL-C, LCI and non-HDL-C easily calculated and were not affected by diet and sex, which unveiled a new way to predict hypothyroidism and SCH's relation of cardiovascular diseases.

Data showed that Hcy may increase the risk of CHD by $4 \%$ for each $4 \mu \mathrm{mol} / \mathrm{L}$ increased; the increase of Hcy $5 \mu \mathrm{mol} / \mathrm{L}$ had the same effect on increasing the incident of CHD with increasing of TC $0.5 \mathrm{mmol} / \mathrm{L}$ [21]. Song Qingzhang et al [9] found that Hcy of patients with hypothyroidism significantly negatively correlated with FT3 and FT4 and significantly positively correlated with TG, and not correlated with TC, HDL-C, and LDL-C; therefore elevated serum of Hcy was believed to be an important risk factor for hypothyroidism complicated with CHD. Sun Jihong et al [22] found that FT3 of patients with hypothyroidism negatively correlated with TC and LDL-C; FT4 negatively correlated with TC, HDL-C, and LDL-C. Yu Wenzhu et al. [23] reported that patients with hypothyroidism and $\mathrm{SCH}$ had significantly elevated TG, TC, LDL-C and Hcy and SCH, which positively correlated with TSH; and HDL-C decreased. Zhou Chunjuan et al [4] found that in the process of transformation from $\mathrm{SCH}$ to hypothyroidism, Hcy and TSH positively correlated with each other. Patients with hypothyroidism with elevating serum of Hcy indicated a higher incidence of cardiovascular diseases; therefore combined detection of TSH and Hcy might be of great value in the diagnosis and treatment of hypothyroidism. Xiong $\mathrm{Lu}$ et al [24] found that slightly elevated TSH $(<10 \mathrm{mIU} / \mathrm{L})$ had little effect on TG and LDL-C, but the plasma Hcy might be increased; and significantly elevated $\mathrm{TSH}(\geq 10 \mathrm{mIU} / \mathrm{L})$ might cause $\mathrm{TG}$, LDL-C, and Hcy to increase significantly. A meta-analysis showed that the level of Hcy associated with the severity of hypothyroidism and that treatment with L-T4 reduced the level of Hcy in patients [25]. This study found that patients with hypothyroidism and TSH had significantly higher serum of Hcy than those in the control group, and there was significant difference between the two groups, which was consistent with the above findings; and TSH is positively correlated with TC, TG, LDL-C, TC/HDL-C, TG/HDL-C, LDL-C/HDL-C, LCI, non-HDL-C and Hcy.

\section{Conclusion}

In general, patients with hypothyroidism are often complicated with significant dyslipidemia and abnormal Hcy, manifested as significantly elevated TG, TC, LDL-C and Hcy; and TG/HDL-C, LDL-C/HDL-C, LCI and non-HDL-C were significantly higher than those in the $\mathrm{SCH}$ and control groups. $\mathrm{SCH}$ patients also underwent changes accordingly in their blood lipid profile, blood lipid ratios and Hcy. The changes in blood lipid profile and blood lipid ratios and elevated Hcy were considered the risk factors of hypothyroidism complicated with CHD. In addition, blood lipid ratios are of significantly high value in prediction than single blood lipid indicator. With the improvement of thyroid functions after treatment, blood lipid profile, blood lipid ratios and Hcy can return to normal levels to prevent the occurrence of CHD. Therefore, dynamic monitoring of blood lipid ratios and Hcy are of great value in the transformation to hypothyroidism and prediction of cardiovascular diseases. Combined detection has unveiled a new way to the prevention and treatment of hypothyroidism complicated with cardiovascular diseases.

\section{Acknowledgements}

This study was supported by a Natural Science Project of Cangzhou Medical College (No.14Z001), a Science and Technology Program Fund Project of Hebei in China (No.15277796D).

\section{References}

[1] Wiles KS, Jarvis S, Nelson-Piercy C. Are we overtreating subclinical hypothyroidism? [J]. BMJ, 2015, vol 351: pp.h4726.

[2] ABULAITI Alimujiang, Li B, ZHang L, et al. Relationship between subclinical hypothyroidism and caronary artery disease [J] Medical Journal of the Chinese People's Armed Police Force, 2016, vol 27, pp.221-224.

[3] Kamasaki H, Takeuchi T, Mikami T, et al. A case of graves' disease diagnosed in the course of bilateral carotid artery stenoses(moyamoya disease ); a case report and review of the literature [J]. Clin Pediatr Endocrinol,2013, vol.22, pp.39-44.

[4] ZHou CJ, ZHu XL, Wang CG, et al.The diagnosis value of combined detection of $\mathrm{TH}$ and Hcy levels for patients with hypothyroidism [J]. Chin J Health Lab Tec, 2016, vol.26,pp.2205-2207.

[5] ZHao JJ, Yang LB. Hypothyroidism and dyslipidemia [J]. Chinese Journal of Practical Internal Medicine Apr 2014, vol.34, pp. $340-343$.

[6] Garduno-Garcia Jde J, Alvirde-Garcia U, Lopez-Carrasco G, et al. TSH and free thyroxine concentrations are associated with differing metabolic markers in euthyroid subjects [J]. Eur J Endocrinol, 2010, vol.163, pp.273-278.

[7] Fu YL, Fa LM. Research Progress on the relationship between thyroid function and metabolic syndrome $[\mathrm{J}]$. J Mod Med Health January, 2017, vol.33, pp.77-79.

[8] Li F. Determination and clinical significance of subclinical hypothyroidism in patients with serum homocysteine and blood lipids [J]. Zhejiang Practical Med, 2009, vol.14, pp.366-367.

[9] Song QZ, Xu FL, Bi XP, et al. Clinical significance of serum homocysteine and lipids determination in hypothyroidism [J]. Lab Med, 2007, vol.22, pp.176-178.

[10] Toruner F, Altinova AE, Karakoc A, et al. Risk factors for cardiovascular disease in patients with subclinical hypothyroidism [J]. Adv Ther, 2008, vol.25, pp.430 437. 
[11] LópezRubio MA, TárragaLópez PJ, RodríguezMontes JA, et al. Subclinical hypothyroidism and cardiovascular risk [J]. Nutr Hosp, 2015, vol.31,pp.2095-2102.

[12] van Tienhoven-Wind LJ, Dullaart RP. Low-normal thyroid function and the pathogenesis of commoncardio-metabolic disorders [J]. EurJClin Invest, 2015, vol.45, pp.494-503.

[13] Karthick N, Dillara K, Poornima KN, et al. Dyslipidaemic changes in women with subclinical hypothyroidism [J]. J Clin Diagn Res, 2013, vol.7, pp.2122-2125.

[14] Laway BA, War FA, Shah S, et al. Alteration of lipid parameters in patients with subclinical hypothyroidism[J]. Int J Endocrinol Metab, 2014, vol.12,pp.e17496.

[15] Díez JJ, Iglesias P. Serum cholesterol and triglyceride concen-trations in diabetic patients with subclinical hypothyroidism [J]. Endocrinol Nutr, 2014, vol.61, pp.419-425.

[16] Wang C, Zhang P. Clinical research progress of lipid ratio in predicting cardiovascular disease $[\mathrm{J}]$. Shandong Medical Journal, 2015, vol.55,pp.99-102.

[17] Huang X, Cui ZW,Wu W, et al. Application of blood lipid ratio in risk assessment of cardiovascular and cerebrovascular diseases [J]. International Journal of Laboratory Medicine, 2015, vol.36, pp.1274-1276.

[18] Xie YJ. The level and significance of serum lipids in patients with different types of coronary heart disease [J]. Contemporary Medicine, 2012, vol.18,pp.10-11.

[19] Simprini LA, Villines TC, Rich M, et al. The relationships between subclinical atherosclerosis, non-high-density lipoprotein cholesterol, exercise and diet among male participants of the PACC project [J]. J Clin Lipidol, 2012, vol.6, pp.174-179.

[20] Chen XJ, Huang ZH. Non high density lipoprotein cholesterol in coronary atherosclerotic heart disease the value of predictive and prognostic assessment [J]. China Medical Engineering, 2016, vol.24, pp.53-55.

[21] Wu ZR, ZHang LR, Pan YQ, Changes and clinical significance of serum homocysteine and lipids in Patients with primary hypothyroidism and hyperthyroid [J]. Progress in Modern Biomedicine, 2009, vol.9,pp.4276-4278.

[22] Sun JH, ZHang Y, Lu HR, et al. Correlation of serum adiponectin with thyroid dysfunction in elderly people $[\mathrm{J}]$. Lab Med, 2016, vol.31, pp.549-552.

[23] Yu WZ, Yuan D, Fan YX, et al. Correlation analysis of serum lipids, homocysteine and serum uric acid in patients with subclinical hypothyroidism [J]. Chinese J Lab diagnosis, 2015, vol.19, pp.1496-1497.

[24] Xiong L, Luo CD, Liu YB, et al.Changes of Plasma Homocysteine and Peripheric Arterial Stiffness in Patients with Subclinical Hypothyroidism [J]. West China Medical Journal, 2015, vol.30, pp.2205-2207.

[25] Zhou Y, Chen Y, Cao X. Association between plasma homocysteine status and hypothyroidism: a meta- analysis [J]. Int J Clin ExpMed, 2014, vol.7, pp.4544-4553.

\section{Biography}

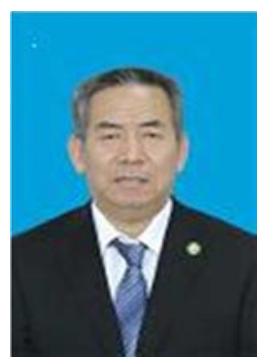

Hou Zhenjiang (1958-), male, with bachelor degree, Director of the Institute of Thyroid Disease, Professor, engaged in medical testing education and scientific research, the Hebei Province Higher Education Famous Teacher, medical and health assessment expert for A Guide to the Core Chinese periodical (2011 and 2014 Edition). 